\title{
Evaluation of High-Performance Polymers and Composites Based on Material and Structural Indices
}

\author{
M. TAKAYANAGI \\ Department of Industrial Chemistry, Faculty of Engineering, \\ Kyushu Sangyo University, \\ Matsukadai, Fukuoka 813, Japan
}

(Received August 17, 1986)

\begin{abstract}
Advanced composite materials such as carbon and aramid fiber-reinforced composite materials, molecular composites, liquid crystal polymers and other super-engineering polymers have been put on the market for special use or in a stage of exploration. They are conceived to be employed in the primary structures of space structure and air planes or the parts of precise electronic instruments due to their high-performance properties. It becomes necessary to evaluate their properties in order to select the appropriate materials for final use. The material and structural indices proposed by Hayashi for the orthotropic materials are suitable for the purpose intended. The larger these values, the more efficient the structural element made of them, that is, the lightest element enduring the mechanical requirements can be selected. The structural indices of present commercialized liquid crystal polymers whether orthotropic or isotropic are somewhat inferior to those of metal alloys. Far excellent are the molecular composites proposed by the U.S. Air Force Material Laboratories, the structural indices of which are comparable to those of carbon fiber/epoxy composites which are proven to be practically useful. The index values of materials including metal alloys are compared in bar graphs.

KEY WORDS Advanced Composite Materials / Material Index / Structural Index / High Performance Polymers /
\end{abstract}

High-performance polymeric materials and advanced composite materials are conceived to be employed as the construction materials of transportation vehicles such as air plane, rocket and car, space structure and the parts of precise electronic instruments. For this purpose, it is effective to employ some indices representing their properties, with which the appropriate selection of new materials is made. Hayashi ${ }^{1}$ calculated several structural indices of orthotropic materials to apply to the fiberreinforced composite materials.

Material and structural indices proposed by Hayashi are similarly applicable to the monolithic orthotropic high-performance polymeric materials. In this paper, the indices of liquid crystal polymers newly appearing in the market and those of molecular composites in a stage of exploration are evaluated and compared with those of fiber composites and metal alloys already reported.

Hayashi discriminated between material and structural indices with whether the size of structural element is taken into account or not. The larger the value of index, the more efficient the structural element is, in the meaning that the lightest weight is achieved by using the material with the largest index value. This requirement is one of the most important properties for use in space and aeronautical fields.

\section{MATERIALS AND MECHANICAL DATA}

\section{Liquid Crystal Polymers}

Jackson and others ${ }^{2}$ published a scientific 
paper in 1976 on thermotropic liquid crystal polymer which is a copolymer of poly(ethylene tetrephthalate) (PET) and $p$-hydroxy benzoic acid (HBA). This paper have given rise to the active research and development for thermotropic liquid crystal polymers with high performance. In a molten state they show nematic phase, which enables injection and extrusion to provide a thin layer with high anisotropy and a solid with novel properties. Eastman Kodak's product of the same species is called X7G. EPE of Mitsubishi Chemical Industries Co. and LC2000 of Yunitica Co. in Japan are considered essentially belonging to $\mathrm{X} 7 \mathrm{G}$.

Homopolymer of HBA was synthesized by Economy about 15 years ago, which was incapable of melting and dissolution. Ekkcel of Carborundum Co. was manufactured by copolymerizing HBA with biphenyl terephthalate to improve the processability. Xydar of Dartco Mfg. Co. was put on the market for the first time in 1984. Injection temperature is $350-400^{\circ} \mathrm{C}$ and heat-distortion temperature is $300-350^{\circ} \mathrm{C}$. Heat-resistance of Xydar is comparable to that of polyimide and superior in fabricability. Ekonol of Sumitomo Chemical
Industries Co. belongs to the series of Carborundum Co. in its constitution, which was proposed as a high-performance fiber comparable to Kevlar aramid fiber. Injection temperature of these polymers is very high so that a special molding machine is demanded.

Vectra of Celanese Co. was put on the market in 1985 by improving the difficulties of processability of Xydar. Polyplastics Co. in Japan sold Vectra at the same time. Chemical structure of Vectra is a copolymer of 2hydroxy-6-naphthoic acid and HBA or 2,6dihydroxy naphthalene terephthalate and HBA.

When the molten liquid crystal is injected through a die, the shear stress at the wall of die is maximum and decreases toward the central axis of die. Molecular orientation follows the shear stress gradient and keeps its tendency in crystalline texture after exiting the die. Thus, the anisotropy in modulus and strength varies with the thickness of molded product. Figure 1 shows the scanning electron micrograph of surface appearance of fractured test specimen of an injection-molded sheet of Xydar. The surface layer is strongly fibrillated due to high orientation of molecular axis. In a paper of

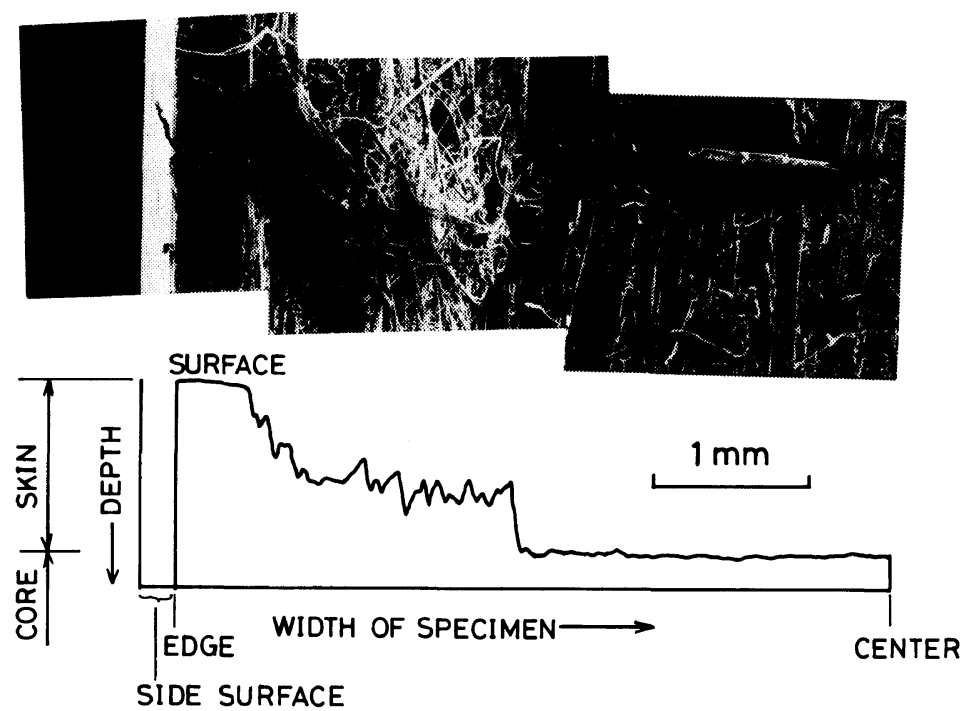

Figure 1. Scanning electron micrograph of surface appearance of fractured test-specimen of injectionmolded sheet of Xydar. 
Table I. Mechanical and density data of liquid crystal polymers

\begin{tabular}{ccccccc}
\hline $\begin{array}{c}\text { Liquid crystal } \\
\text { polymers }\end{array}$ & Orientation & $E_{\mathrm{L}} / \mathrm{GPa}$ & $E_{\mathrm{T}} / \mathrm{GPa}$ & $F_{\mathrm{L}} / \mathrm{MPa}$ & $F_{\mathrm{T}} / \mathrm{MPa}$ & $\rho / \mathrm{gcm}^{-3}$ \\
\hline X7G & Uni- $^{\mathrm{a}}$ & 54.1 & 1.38 & 151 & 10.3 & 1.285 \\
& Iso- $^{\mathrm{b}}$ & 2.21 & - & 63 & - & 1.40 \\
\hline Vectra & Uni- & 10.6 & 2.6 & 144 & - & - \\
\hline Xydar & Iso- & 5.0 & - & 138 & - & 1.35 \\
\hline
\end{tabular}

${ }^{a}$ Uni- means unidirectionally oriented material.

b Iso- means isotropic material or $E_{\mathrm{L}}=E_{\mathrm{T}}$ and $F_{\mathrm{L}}=F_{\mathrm{T}}$.

Jackson and others, ${ }^{2}$ the bending modulus and bending strength are represented as a function of thickness of injected sheet. At sufficiently large thickness the data along and across the molecular orientation accord and the anisotropy almost disappears, in which case crystal orientation is statistically random in bulk except for highly oriented surface layer. If the data are extrapolated to zero thickness, the modulus and strength of unidirectionally oriented specimen along and across the orientation are evaluated, which are denoted by $E_{\mathrm{L}}$ and $E_{\mathrm{T}}$ for modulus and $F_{\mathrm{L}}$ and. $F_{\mathrm{T}}$ for strength, respectively. The density of the specimen is denoted by $\rho \mathrm{g} \mathrm{cm}^{-3}$. Table I lists the data available from a paper ${ }^{2}$ and the corresponding catalogues.

\section{Molecular Composites}

Molecular composites are prepared by dispersing rigid rod molecules in the matrix of thermotropic or thermosetting resin. This material is in a stage of exploration and of scientific interest.

The group of the U.S. Air Force Wright Aeronautical Laboratories and Dayton Research Institute reported a molecular composite, which is composed of poly ( $p$-phenylene benzobisthiazole) (PBT) as reinforcing rigid rod molecule and flexible poly(2,5(6)benzimidazole) (ABPBI) as a matrix polymer. ${ }^{3}$ According to the literature, a spinning dope of strong acid solution of both components is stretched at the time of coagulation, with which molecular dispersion is realized. For the sheet of 30/70-PBT/ABPBI, the following data are obtained.

$$
\begin{array}{ll}
E_{\mathrm{L}}=120.3 \mathrm{GPa}, & E_{\mathrm{T}}=16.8 \mathrm{GPa}, \\
F_{\mathrm{L}}=1.508 \mathrm{GPa}, & F_{\mathrm{T}}=0.675 \mathrm{GPa},
\end{array}
$$

and

$$
\rho=1.55 \mathrm{~g} \mathrm{~cm}^{-3} .
$$

Personal information on the in-plane isotropic sheet provided the following data.

$$
E_{\mathrm{L}}=E_{\mathrm{T}}=62 \mathrm{GPa} \text { and } F_{\mathrm{L}}=F_{\mathrm{T}}=0.7 \mathrm{GPa} \text {. }
$$

It will be instructive to cite another type of molecular composite with in-plane isotropic sheet having the following data.

$$
E_{\mathrm{L}}=E_{\mathrm{T}}=60 \mathrm{GPa} \text { and } F_{\mathrm{L}}=F_{\mathrm{T}}=0.5 \mathrm{GPa} \text {. }
$$

This sheet was prepared in my laboratory by using a solution of poly( $p$-phenylene terephthalamide) (PPTA) reacted with epoxy resin and a resin of bismaleimide and diamine (Kerimid of Rhone Poulinc Co.), being followed by solvent-cast and cure. $^{4}$ The best record is cited here. The reproducibility is not satisfactory and appropriate to assume this specimen to be fictitious. The sample is denoted by RRM/BMI. 
Table II. Mechanical and density data of fiber reinforced composite materials

\begin{tabular}{|c|c|c|c|c|c|c|c|c|}
\hline $\begin{array}{c}\text { Fiber } \\
\text { composite }\end{array}$ & Orientation & $E_{\mathrm{L}} / \mathrm{GPa}$ & $E_{\mathrm{T}} / \mathrm{GPa}$ & $F_{\mathrm{L}} / \mathrm{GPa}$ & $F_{\mathrm{T}} / \mathrm{GPa}$ & $v_{\mathrm{LT}}^{\mathrm{c}}$ & $v_{\mathrm{TL}}^{\mathrm{c}}$ & $\rho / \mathrm{g} \mathrm{cm}^{-3}$ \\
\hline GF/EPOXY ${ }^{5}$ & Uni- $^{a}$ & 43 & 11.6 & 1.2 & 0.04 & 0.29 & 0.083 & $2.0\left(V_{\mathrm{f}}=55 \%\right)$ \\
\hline CF/EPOXY & $\begin{array}{l}\text { Uni- }{ }^{5} \\
\text { Pseudoiso-6,b }\end{array}$ & $\begin{array}{r}151 \\
52\end{array}$ & $\begin{array}{l}9.9 \\
-\end{array}$ & $\begin{array}{l}1.9 \\
0.56\end{array}$ & $\frac{0.068}{-}$ & $\begin{array}{c}0.31 \\
-\end{array}$ & $\begin{array}{c}0.022 \\
-\end{array}$ & $\begin{array}{l}1.5\left(V_{\mathrm{f}}=60 \%\right) \\
1.6\end{array}$ \\
\hline $\mathrm{AF} / \mathrm{EPOXY}^{5}$ & Uni- & 77 & 5.6 & 1.4 & 0.03 & - & - & $1.38\left(V_{\mathrm{f}}=60 \%\right)$ \\
\hline
\end{tabular}

\section{Fiber-Reinforced Composite Materials}

Fiber-reinforced metal (FRM) and fiberreinforced plastics (FRP) are both leading composite materials, although recently hybrid composites such as aluminum plate and resin appeared. Fiber composites of organic origin are represented by carbon fiber (CF)/epoxy and Kevlar aramid fiber (AF)/epoxy. Traditional glass fiber (GF)/epoxy is also used if necessary. Aluminum alloy used in the primary structure of air plane is, for example, being gradually replaced by $\mathrm{CF} / \mathrm{epoxy}, \mathrm{AF} /$ epoxy or their hybrid composites.

There are many published data on these materials. Arbitrarily the data are taken from the following literature and summarized in Table II. The data of GF/epoxy are taken from Ohira's recent measurements, ${ }^{5}$ the data of CF/epoxy are obtained from Norita's paper $^{6}$ on pseudoisotropic composites and from Ohira's paper ${ }^{5}$ on unidirectionally reinforced composites, and the data of AF/epoxy are taken from Norita's article. ${ }^{6}$

\section{MATERIAL AND STRUCTURAL INDICES}

\section{Material Indices-Specific Strength and Specific Modulus}

The column made of material i with arbitrary cross-sectional shape and length $l$ is assumed to endure the tensile force $P$. The density of the material is denoted by $\rho_{\mathrm{i}}$, tensile strength by $\sigma_{\mathrm{ui}}$, the cross-sectional area by $A_{\mathrm{i}}$ and the total weight by $W_{\mathrm{i}}$. The cross-sectional area $A_{\mathrm{i}}$ is $P / \sigma_{\mathrm{ui}}$. Thus, the weight of the element is

$$
W_{\mathrm{i}}=\rho_{\mathrm{i}} A_{\mathrm{i}} l=\frac{\rho_{\mathrm{i}} l P}{\sigma_{\mathrm{ui}}}
$$

According to this equation, the larger the value of $\sigma_{\mathrm{ui}} / \rho_{\mathrm{i}}$, the more efficient the material is, that is, the lightest structural element is realized with the requested function. $\sigma_{\mathrm{ui}} / \rho$ is called specific strength. In the case of fracture by compressive force, $\sigma_{\mathrm{u}}$ is replaced by compressive strength. In the case of fracture by shear stress, $\sigma_{u}$ is replaced by shear strength $\tau_{\text {ui }}$.

When the tensile strain $\varepsilon$ is given beforehand, $A_{\mathrm{i}}$ is given by $P / \sigma_{\mathrm{i}}=P / \varepsilon E_{\mathrm{i}}$ where $E_{\mathrm{i}}$ is the tensile modulus of the material. Thus, $W_{\mathrm{i}}$ is given by $P l \rho_{\mathrm{i}} / \varepsilon E_{\mathrm{i}}$. According to this relation, the larger the value of $E_{\mathrm{i}} / \rho_{\mathrm{i}}$, the more efficient the material is for requested stiffness of the element. In the case of shear stiffness, $E_{\mathrm{i}}$ is replaced by $G_{\mathbf{i}}$.

These indices are termed by Hayashi ${ }^{1}$ as the material indices, which are essentially irrespective of the element size. On the other hand, the structural indices mentioned in the next section are related to some given size of the element. 


\section{Structural Indices}

A structure is constructed from column, cylinder and plate. Each element is exerted by tensile, compressive, and shearing forces depending on design. First, the buckling of column by compressive force will be considered from the view point of selecting the most efficient material providing the lightest weight. Euler's equation gives the critical load at which the column suddenly shows buckling phenomenon.

$$
P_{\mathrm{cr}}=C \frac{\pi^{2} E_{\mathrm{i}} I_{\mathrm{i}}}{l^{2}}
$$

where $P_{\mathrm{cr}}$ is the critical load, $l$ is a given length of column, $E_{\mathrm{i}}$ is the tensile modulus of the material $\mathrm{i}$, and $I_{\mathrm{i}}$ is the second moment of the element. $C$ is the buckling coefficient: $C=1$ for the column with both simply supported ends (SS), $C=4$ for the column with both clamped ends (CL), and $C=2.0458$ for the column with $S$ and $C L$ ends.

Application of eq 2 to the buckling of a column with circular cross-section with diam- eter $d_{\mathrm{i}}$ gives the following results. $I_{\mathrm{i}}$ of the column with circular cross-section is $\pi d_{\mathrm{i}}^{4} / 64$, which value is substituted into eq 2 . Rewriting of the equation results in

$$
d_{\mathrm{i}}=\left(64 l^{2} / \pi^{3} C E_{\mathrm{i}}\right)^{1 / 4} .
$$

Thus, the total weight of the element $W_{\mathrm{i}}$ is

$$
\begin{aligned}
W_{\mathrm{i}} & =\frac{\pi}{4} l d_{\mathrm{i}}^{2} \rho_{\mathrm{i}}=\frac{\pi}{4} l \rho_{\mathrm{i}}\left(64 l^{2} / \pi^{3} C E_{\mathrm{i}}\right)^{1 / 2} \\
& =K /\left(\sqrt{E_{\mathrm{i}}} / \rho_{\mathrm{i}}\right)
\end{aligned}
$$

where $K$ is composed of the given values. Thus, the larger the value of $\sqrt{E_{\mathrm{i}}} / \rho_{\mathrm{i}}$, the more efficient the structural element with circular cross-section. The lightest weight of the element is achieved by using such a material i. If the column has a rectangular cross-section with given width $b$ and thickness $t_{\mathrm{i}}$ (variable), then the second moment is $b t_{\mathrm{i}}^{3} / 12$ and the total weight $W_{\mathrm{i}}$ is represented by $K^{\prime} /\left(\sqrt[3]{E_{\mathrm{i}}} / \rho_{\mathrm{i}}\right)$. Thus, the structural index of the column with rectangular cross-section is $\sqrt[3]{E_{\mathrm{i}}} / \rho_{\mathrm{i}}$.

The fracture of the column with circular

Table III. Structural indices of structural elements ${ }^{1}$

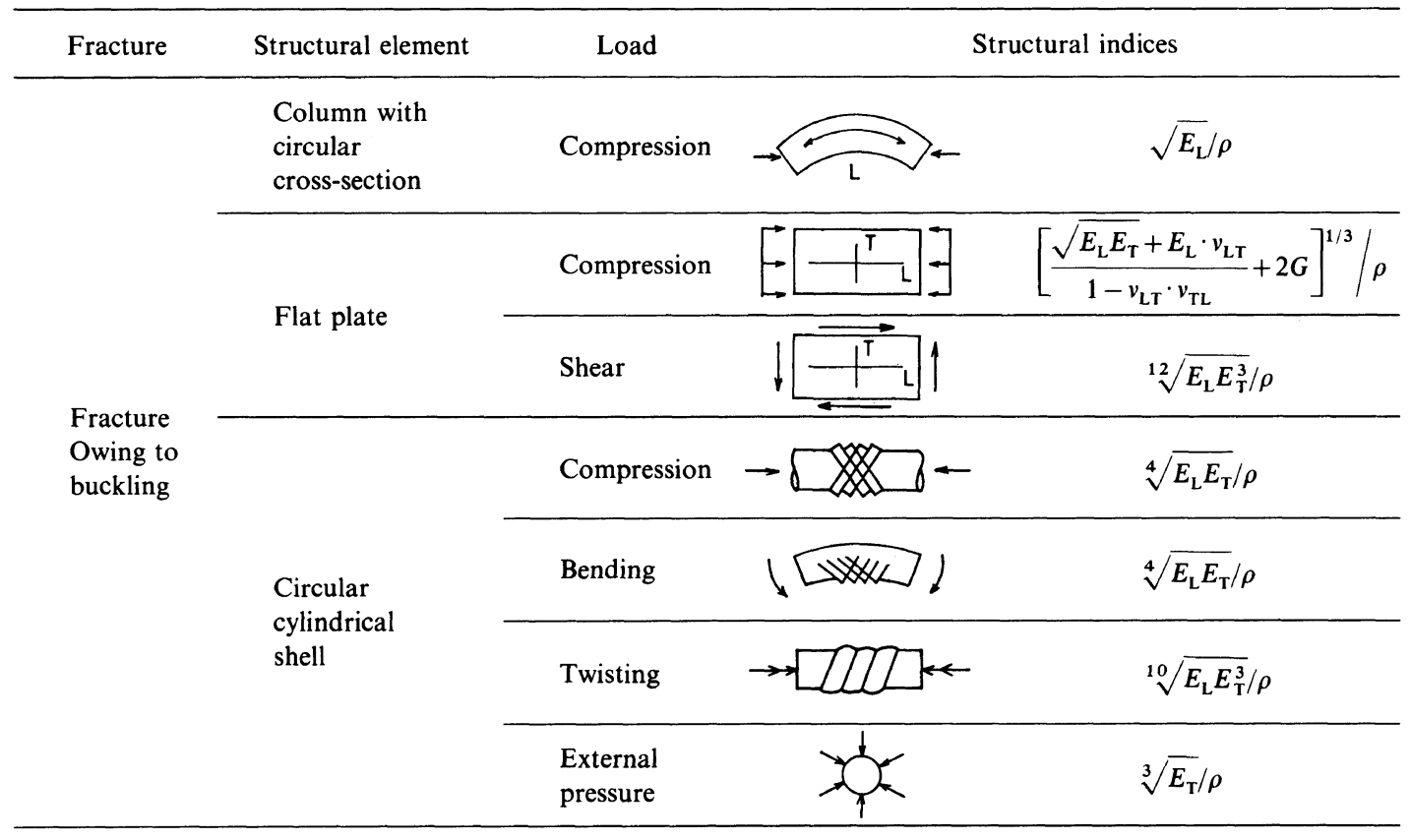


cross-section with diameter $d_{\mathrm{i}}$ by bending moment $M$ is represented by the following equation using the maximum bending stress $\sigma_{\text {Bui }}$.

$$
d_{\mathrm{i}}=\left(32 M / \pi \sigma_{\mathrm{Bui}}\right)^{1 / 3}
$$

Thus, the total weight of the column with circular cross-section, $W_{\mathrm{i}}$ is

$$
\begin{aligned}
W_{\mathrm{i}} & =\frac{\pi}{4} d_{\mathrm{i}}^{2} l \rho_{\mathrm{i}}=\frac{\pi}{4} l \rho_{\mathrm{i}}\left(32 M / \sigma_{\mathrm{Bui}}\right)^{2 / 3} \\
& =K^{\prime \prime}\left(\sqrt[\frac{2}{3}]{\sigma_{\mathrm{Bui}}} / \rho_{\mathrm{i}}\right)
\end{aligned}
$$

Thus, the larger the value of $\sqrt[2 / 3]{\sigma_{\mathrm{Bui}}} / \rho_{\mathrm{i}}$, the more efficient the column with circular crosssection is for bending fracture. The structural index for stiffness of the column with circular cross-section is evaluated to the given curvature in bending by $\sqrt{E_{\mathrm{i}}} / \rho_{\mathrm{i}}$.

Hayashi ${ }^{1}$ achieved the derivation of structural indices in more complicated cases of orthotropic materials with $E_{\mathrm{L}}$ along and $E_{\mathrm{T}}$ across the orientation direction. Table III summarizes some of his derivation. In the case of circular cylindrical shell, only the indices for given shell thickness and length in addition to the given values of compressive force $P$, bending moment $M$, twisting torque $T$ or external side pressure $p$ are arbitrarily adopted. For isotropic plate and shell, $E_{\mathrm{L}}$ is equal to $E_{\mathrm{T}}$.

\section{COMPARISON OF MATERIAL AND STRUCTURAL INDICES OF VARIOUS MATERIALS}

\section{Material Selection Based on Material Indices}

In order to achieve the structure of light weight and high tensile strength, the materials having large strength and modulus per unit weight must be selected. For this purpose, the location of the materials in a map of specific strength versus specific modulus is useful in material selection.

Figure 2 shows such a map, in which the existing materials such as inorganic fiberreinforced metals (FRM), metal alloys such as titan, aluminum and magnesium alloys and
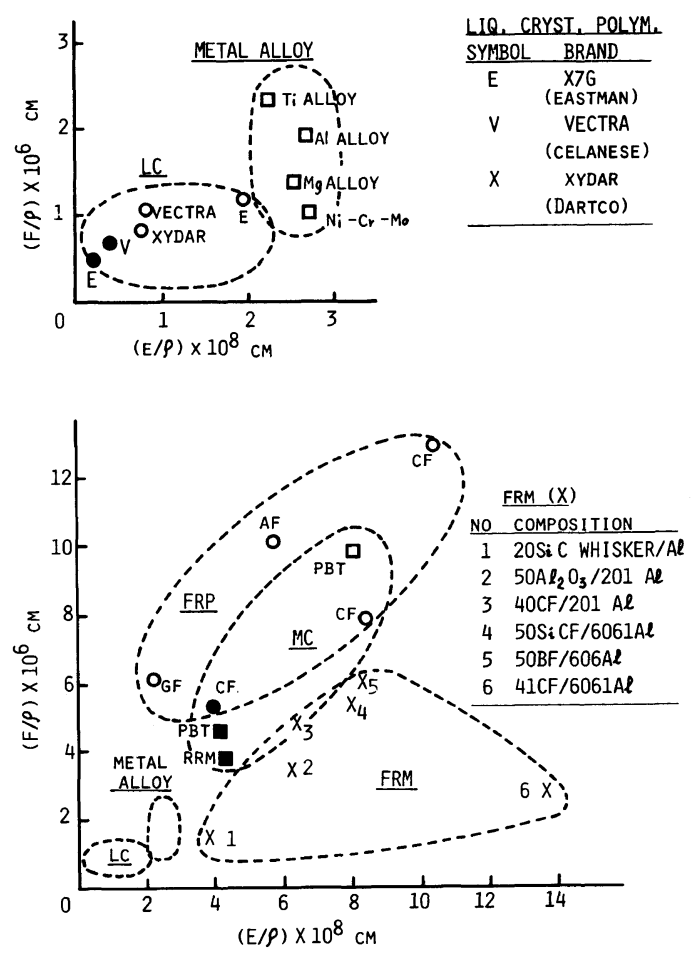

Figure 2. Specific strength $v s$. specific modulus of various materials. Open circles and squares are for unidirectionally anisotropic materials and filled circles and squares are for isotropic and pseudo-isotropic materials. Units of both axes are $\mathrm{cm}$.

fiber-reinforced plastice (FRP) are located. The anisotropy of metal alloys is unnecessary to take into account. Uni-directionally reinforced FRP is represented by open circles, and the pseudoisotropic FRP laminates composed of $0^{\circ}, \pm 45^{\circ}$ and $90^{\circ}$ prepregs are indicated by filled circles. The data of metal alloys and FRM are taken from a review article of Kikukawa. $^{7}$ The oriented and isotropic molecular composites of 30/70-PBT/ABPBI are discriminated by open and filled squares, respectively. The data of RRM/BMI are essentially isotropic. Liquid crystal polymers of X7G, Vectra and Xydar are also plotted. Each group is encircled by broken lines for easy discrimination. Units of modulus and strength are $\mathrm{kg} \mathrm{cm}^{-2}$ and that of density is $\mathrm{kg} \mathrm{cm}^{-3}$, resulting in the units of both coordinate axes being $\mathrm{cm}$. 
When compared the metal alloys with FRP and FRM, the latter groups are superior to metal alloy group. FRP is excellent in specific strength and FRM in specific modulus.

Liquid crystal polymers (LC) presently commercialized are inferior to metal alloys but it can not be concluded as there are many possibilities in LC to be explored for the future. Fabrication method utilized in LC is far excellent compared with that of FRP, in which cautious handling of prepregs is asked.

Molecular composite are in a stage of exploration but their performance is comparable to that of FRP. Isotropic sheet of MC is almost equal to pseudoisotropic CFRP in their locations in a map.

When the CFRP has been already proven to be useful in practical use in the space and aeronautical fields, the criterion for newly appearing advanced polymeric materials should satisfy the following both conditions at the same time.

$$
(E / \rho) \times 10^{8} \mathrm{~cm}>5 \text { and }(F / \rho) \times 10^{6} \mathrm{~cm}>5 .
$$

\section{Material Selection Based on Structural Indices}

Structural indices cited in Table III are evaluated by substituting the data in Table I, II, and the values cited at the molecular composites. Figure 3 shows the comparison of the indices of the column with circular crosssection of various materials for the buckling by the compressive force. The unit of $E$ is kgf $\mathrm{mm}^{-2}$ and the density $\rho$ is $\mathrm{g} \mathrm{cm}^{-3}$. FRP and $\mathrm{MC}$ are superior to aluminum alloy and LC. There is no remarkable difference between metal and LC. It should be noted that LC is injection-moldable and the construction process will be more simplified compared with FRP. The target of high-performance polymeric materials in this case should be $\sqrt{E} / \rho>50$, judging from the data of practically proven CFRP.

The structural index of flat plate for buckling by compressive force is given in Table III by Hayashi. ${ }^{1}$ It is necessary to know the data

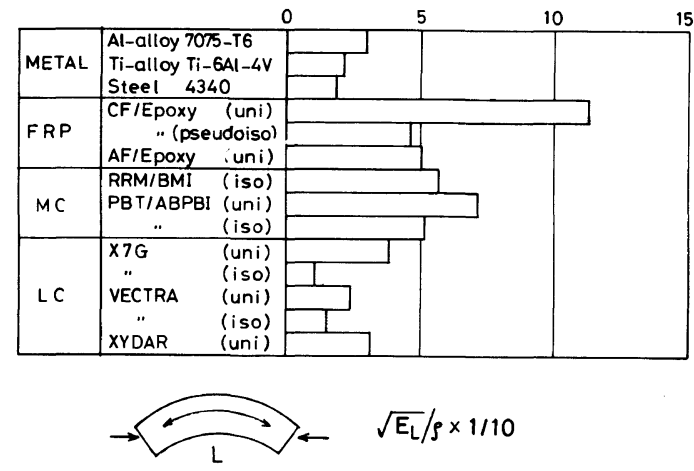

Figure 3. Comparison of structural indices for the buckling of various materials by compressive force. Unit of $E$ is $\mathrm{kgf} \mathrm{mm}^{-2}$.

of tensile modulus, $E$, Poisson's ratio $v$, and shear modulus $G$ along and across the orientation direction of the orthotropic materials. For the data of Ohira on CFRP, $G=5.5 \mathrm{GPa}^{7}$ is supplemented. Poisson's ratio of $\mathrm{MC}$ is assumed 0.4 and $G$ is evaluated by $E / 2(1+v)$ for FRM/BMI. For LC, $v$ is assumed 0.3 and $G$ is assumed $2.0 \mathrm{GPa}$. In general, the structural index in this case is difficult to evaluate and Figure 4 shows several results. Here again FRP and MC are superior to metal and LC. It is desirable for the index being larger than 15 .

The structural index of flat plate for the buckling by shear stress is $\sqrt[12]{E_{\mathrm{L}} E_{\mathrm{T}}^{3}} / \rho$. The comparison of the index among various materials is shown in Figure 5. The index includes the cube of transverse modulus compared with the single power index of the longitudinal modulus. This means that the modulus anisotropy is very influential. Figure 5 shows the index value of iso- or pseudo-isotropic materials giving fairly large values with respect to the unidirectionally reinforced ones, and the variation among different types of materials being not so large. Referring to the practically proven CFRP, the index is desirable to be larger than 10.

Figure 6 shows the structural index of circular cylindrical shell for both cases of the buckling by compressive and bending forces. The same formulae are applied to both cases. 

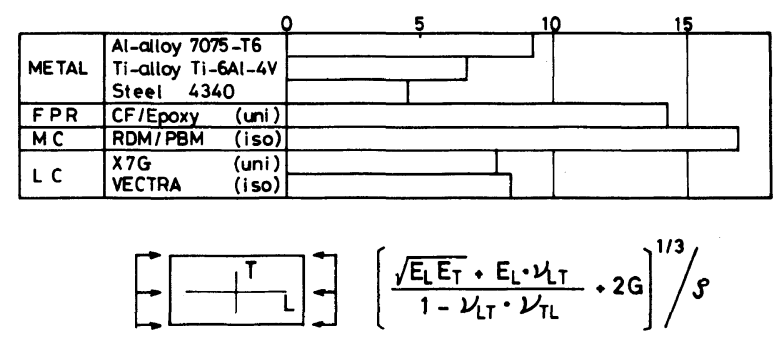

Figure 4. Comparison of structural indices for the buckling of flat plate by compressive force.

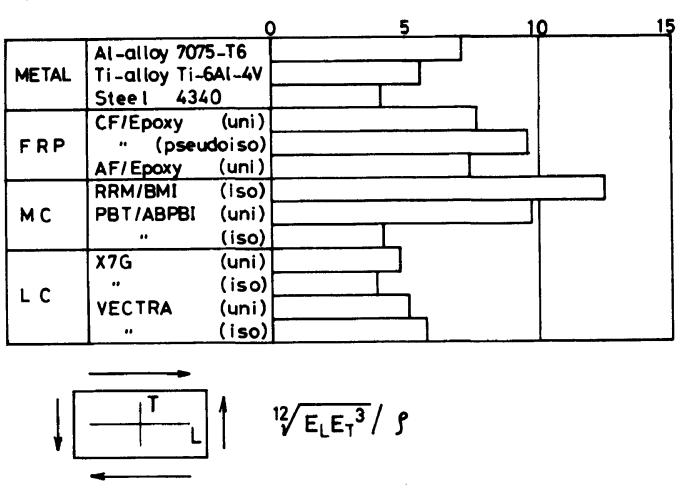

Figure 5. Comparison of structural indices for the buckling of flat plate by shearing force.

The bar in Figure 6 is reduced to one tenth of the calculated value. FRP and MC are superior to metal and LC. The target of the index in this case should be larger than 50 .

Figure 7 shows the comparison of the structural index of circular cylindrical shell for the buckling by twisting torque. The index of $\sqrt[10]{E_{\mathrm{L}} E_{\mathrm{T}}^{3}} / \rho$ includes the cube of transverse modulus and the modulus anisotropy is very influential as in shear buckling of flat plate. Isotropic or pseudoisotropic cylindrical shell is preferable to the unidirectionally oriented one. Again the criterion for buckling of cylindrical shell by twisting is the index being larger than 20. This means that the modulus of isotropic material is larger than $49.3 \mathrm{GPa}$, which value clears almost the criteria for the cases of compression or twisting of cylindrical shell, shear buckling of flat plate, and buckling of column with circular cross-section. To prepare the isotropic plate with moduli of $50 \mathrm{GPa}$

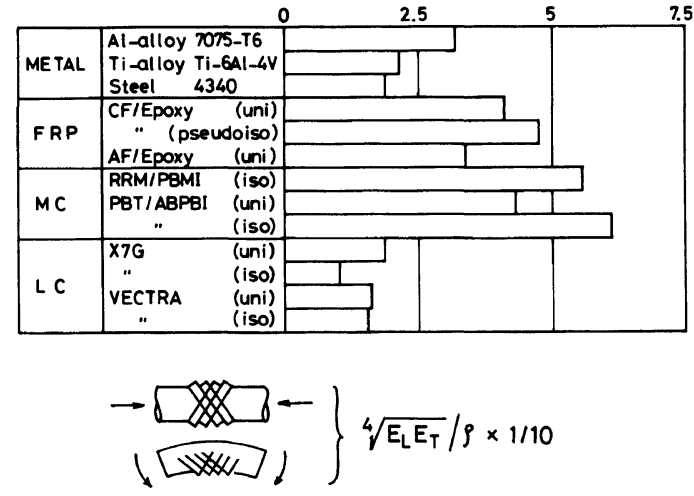

Figure 6. Comparison of structural indices for the buckling of circular cylindrical shell by compressive force or bending moment. Scale of index is shortened to one tenth.

along all directions becomes a good target for the structural element to employ for constructing the structure, for example, in space. Specific modulus associated with tensile strain in this case is, however, somewhat lower than the one indicated in Figure 2.

Figure 8 shows the comparison of the structural indices of circular cylindrical shell for the axis-symmetrical buckling by side pressure. The structural index in this case is only related to the transverse modulus and the merits are found in the case of pseudoisotropic or isotropic specimens of FRP and MC. The index is desirable to be larger than 10 , referring to the data of the pseudoisotropic CFRP.

The structure is constructed from the elements such as column, plate and cylinder. Depending on the design of structure, the stress structure in elements varies from parts to parts. The optimized design of structure gives 

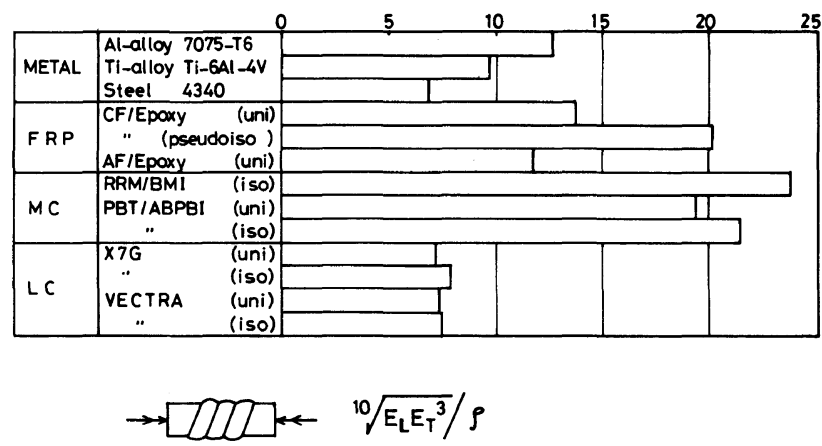

Figure 7. Comparison of structural indices for the buckling of circular cylindrical shell by twisting.

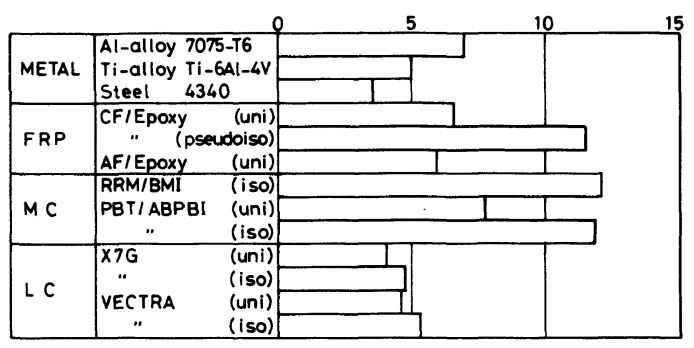

$$
\overbrace{T}^{1} \sqrt[3]{E_{T}} / \rho
$$

Figure 8. Comparison of structural indices for the axis-symmetrical buckling of circular cylindrical shell by side pressure.

the best solution.

\section{CONCLUSION}

Referring to the evaluated values of the material and structural indices, FRP and MC are superior to metal alloy. Liquid crystal polymers have been just put on the market and the data employed here are not necessarily the highest values. They have an advantage in the process of fabrication of parts compared with FRP. The target values of structural indices are summarized as follows. It is not necessary to satisfy all the criterion values at the same time. Especially, the element enduring tension and the one enduring buckling fracture may be discriminated, especially when the optimization of structure design is made.

\section{Criteria:}

(1) Specific modulus $(E / \rho) \times 10^{8} \mathrm{~cm}>5$

(2) Specific strength $(F / \rho) \times 10^{6} \mathrm{~cm}>5$

(3) Buckling of flat plate by compression

$$
\left[\frac{\sqrt{E_{\mathrm{L}} E_{\mathrm{T}}}+E_{\mathrm{L}} v_{\mathrm{LT}}}{l-v_{\mathrm{LT}} v_{\mathrm{TL}}}+2 G\right]^{1 / 3} / \rho>15
$$

(4) Buckling of column with circular cross-section by compressive force

$$
\sqrt{E_{\mathrm{L}}} / \rho>50
$$

(5) Buckling of flat plate by shear

$$
\sqrt[12]{E_{\mathrm{L}} E_{\mathrm{T}}^{3}} / \rho>10
$$

(6) Buckling of cylindrical shell by compression

$$
\sqrt[4]{E_{\mathrm{L}} E_{\mathrm{T}}} \rho>50
$$

(7) Buckling of cylindrical shell by twisting

$$
\sqrt[10]{E_{\mathrm{L}} E_{\mathrm{T}}^{3}} / \rho>20
$$

(8) Axis-symmetrical buckling of cylindrical shell by side pressure

$$
\sqrt[3]{E_{\mathrm{T}}} / \rho>10 \text {. }
$$

Acknowledgement. In the preparation of this article, I highly appreciate the kind support of Prof. H. Ohira of Department of Aeronautical Engineering, Kyushu University, to derive the formulae of indices. 


\section{TAKAYANAGI}

\section{REFERENCES}

1. T. Hayashi, Nippon-Kikai-Gakkaishi, 76, 713 (1973).

2. W. J. Jackson, Jr. and J. R. Kuhfuss, J. Polym. Sci., Polym. Chem. Ed., 14, 2043 (1976).

3. W.-F. Hwang, D. R. Wiff, C. L. Benner, and T. E.
Helminiak, J. Macromol. Sci.-Phys., B22, 231 (1983).

4. M. Takayanagi and S. Ueta, unpublished work.

5. H. Ohira, Kogyo-Zairyo, 32, 43 (1984).

6. T. Norita, Kobunshi, 34, 910 (1985).

7. H. Kikukawa, from "Development and Assessment Technique of Advanced Composite Materials," CMC Co., 1985, p 206. 